\title{
eJRIEPS
}

Ejournal de la recherche sur l'intervention en éducation physique et sport

Hors-série $N^{\circ} 2 \mid 2018$

À propos de certaines bases théoriques et pratiques des sports collectifs

\section{Le temps, le mouvement et la compréhension de l'organisation du jeu}

Jean-Francis Gréhaigne

\section{OpenEdition \\ 12 Journals}

Édition électronique

URL : http://journals.openedition.org/ejrieps/506

DOI : $10.4000 /$ ejrieps.506

ISSN : 2105-0821

Éditeur

ELLIADD

Référence électronique

Jean-Francis Gréhaigne, «Le temps, le mouvement et la compréhension de l'organisation du jeu », eJRIEPS [En ligne], Hors-série № 2 | 2018, mis en ligne le 01 juillet 2018, consulté le 01 août 2019. URL : http://journals.openedition.org/ejrieps/506 ; DOI : 10.4000/ejrieps.506

La revue eJRIEPS est mise à disposition selon les termes de la Creative Commons Attribution 4.0 International License. 


\section{Le temps, le mouvement et la compréhension de l'organisation du jeu}

En sport collectif, dans le mouvement général ou dans la trame dynamique de transformation que cela constitue, les différentes séquences de jeu s'enchaînent. Étudier le jeu en mouvement, c'est concevoir que les actions de jeu sont insérées dans des séquences de jeu mais, en même temps, engendrent cette action. Jouer aux sports collectifs, c'est organiser un mouvement continuel du ballon et des joueurs tour à tour attaquants et défenseurs, dans un va-et-vient entre initiative personnelle et tactique collective. Le mouvement dans le jeu naît, à l'évidence, d'une cohérence entre les joueurs dont la base est l'intelligence tactique et la compréhension. Aussi, quel que soit le jeu sportif collectif considéré, une analyse de la dynamique du jeu (Gréhaigne \& Godbout, 2012) doit aider à rendre compte du rapport de forces global et des rapports de forces partiels entre les joueurs des deux équipes. Ici, le renversement conceptuel en regard d'une approche traditionnelle techniciste consiste bien à considérer que rien ne peut être expliqué ni réalisé sans une bonne compréhension des rapports d'opposition liant constamment les deux équipes au fil du déroulement du jeu. Analyser, expliciter et évaluer l'opposition afin d'utiliser celle-ci comme source de tout progrès, constitue une matrice de références tout en permettant de faire jouer la réalité des forces en présence. Deleplace (1979) affirmait déjà, «nous avons été conduits à démontrer qu'il était nécessaire d'analyser les rapports d'opposition minutieusement et qu'il était parfaitement possible de parvenir à les expliciter, à les formuler et à les systématiser pour leur mise en cuvre consciente et méthodique tant dans l'entraînement ou l'initiation que dans le jeu lui-même » (1979, p. 9). Aujourd'hui, tout le monde est à peu près d'accord pour convenir que pour bien analyser les sports collectifs, il faut recourir à une approche de la dynamique de l'affrontement pour comprendre l'organisation du jeu. Dans les actions de jeu, comme dans beaucoup de phénomènes où les lois de la physique non linéaire s'appliquent, des perturbations apparaissent à partir d'une certaine vitesse du jeu. Il semble exister un seuil critique jusqu'où une vitesse du jeu est tolérable et, tout à coup, l'adaptation disparaît. En un mot, le jeu semble entrer dans un état d'instabilité. Cependant, à un bon niveau, la vitesse dans le jeu en mouvement produit un désordre apparent mais qui renferme une homogénéité particulière autre que la simple répartition spatiale de l'emplacement des joueurs sur le terrain à un instant donné. Il s'agit d'une distribution sur des niveaux énergétiques liés aux vitesses, aux accélérations et / ou décélérations des joueurs ainsi qu'à la vitesse et aux trajectoires du ballon. Celles-ci aboutissent à ce que des états spatialement non homogènes peuvent être pondérés et stabilisés par des distributions qui semblent hétérogènes mais apparaissant comme homogènes si l'on analyse les déplacements et les vitesses réciproques des joueurs et de la balle. 


\section{Le temps, l'espace et le mouvement}

Le jeu en mouvement crée de la dynamique en fonction de la distribution des joueurs sur le terrain et des rapports de vitesse entre ceux-ci (Gréhaigne, Godbout, \& Bouthier, 1997). Une façon de représenter les choses consiste à définir les micro-états du système attaque / défense avec, pour chaque micro-état, l'analyse de la distribution des joueurs sur le champ de jeu en fonction de trois paramètres : leurs positions, leurs orientations et leurs vitesses de déplacement. Cela renvoie à une configuration dynamique temporairement stabilisée qui permet d'anticiper l'évolution du rapport de forces. Face aux phénomènes temporels, la complexité repose ici sur le temps disponible et la vitesse du jeu pour décider et faire. Temps et vitesse apparaissent comme des éléments cruciaux de la réussite et le nécessaire cadre de référence à toute analyse sérieuse.

Un autre aspect du jeu semble intéressant à explorer du point de vue de l'organisation du jeu : il concerne les transitions. Les transitions et le jeu de transition renvoient souvent à des configurations où l'on a un peu de temps pour agir même si la densité des joueurs est moins importante : l'attaque doit conserver son avance éventuelle et la défense doit revenir en barrage ou y rester. Le recours au jeu long et / ou court rapide transforme rapidement les configurations. Dès que le jeu long est possible, les configurations changent de forme et présentent, le plus souvent, un jeu en expansion. Le modèle en compression se retrouve fréquemment dans un jeu sur espace stabilisé avec une densité importante de joueurs. De cette façon, dans ce jeu en mouvement se succèdent des actions de jeu qui relèvent soit d'une concentration, soit d'une dispersion des joueurs. Ainsi, la notion de densité renverrait plutôt à un aspect qualitatif car elle dépend étroitement de la position des joueurs qui sont engagés dans une action dans un environnement donné. La concentration / dispersion semble plus liée à une approche quantitative en terme de nombre de joueurs engagés dans l'action en cours dans un espace particulier. Compression / expansion pour le mouvement et concentration / dispersion à propos d'états statiques successifs (ou aspects temporels discontinus) fonctionnent en étroite symbiose pour qui veut décrypter et anticiper le mouvement dans le jeu en vue de prendre des décisions judicieuses. Ce qui différencie et unifie les notions d'espace et de temps dans une même conception, c'est que la mesure de distance peut être transformée en mesure de temps. C'est en ce sens que l'on pourrait dire que l'espace c'est du temps ! Il est courant de souligner la difficulté de penser le mouvement, le temps en les faisant coexister avec une pensée préalable en terme d'espace. Parfois, notre compréhension est mal à l'aise devant le mouvement. Elle a besoin de l'arrêter, de le fixer, de le rendre ponctuel, avec les contradictions qui s'ensuivent. Dans le jeu, relater qu'une balle est quelque part sur sa trajectoire, c'est l'arrêter. C'est d'une certaine manière une « négation » du mouvement... Alors, on pourrait dire que l'espace est transcendé et traversé par le temps qui en transforme jusqu'à sa forme. Cette conception est celle d'un espace tordu, déformé, courbé pour en donner une image dynamique particulière. Cette approche utilisée au football et en 
sports collectifs permet de comprendre comment un espace topologique, organisé et plat en prenant en compte les positions de joueurs et les trajets et trajectoires de balle, est traversé et transformé par des vitesses, des accélérations et décélérations, des changements de direction. Bergson (1922) défendait l'idée que notre intelligence comprend le temps à partir de l'instant ponctuel : elle le spatialise en quelque sorte, puisque cet état momentané n'est pas une détermination temporelle, mais spatiale. Le temps serait alors la succession de différents instants, comme la ligne est une succession de points. Ici, notre intelligence comprend donc le temps à partir de l'espace et comprendre, ainsi le temps, c'est quand même le détruire comme temps. Dans le jeu, nous sommes souvent dans une succession d'états et c'est essentiellement la mobilité et les déplacements des joueurs qui, mettant en perspective la continuité et la durée de l'action, réintègrent le temps dans sa dimension première.

\section{Espaces, intervalle et espace libre}

Dans les jeux, le rapport au temps est donc premier par rapport à l'espace, même si des aspects spatiaux, comme système de traduction du temps, semblent utiles à certains moments du développement du joueur. Ainsi, lorsque l'on enseigne les sports collectifs, il faut construire des situations adaptées soulignant les contraintes temporelles. La complexification des situations va toujours dans le sens qu'il est nécessaire d'avoir beaucoup de temps pour exécuter les tâches, à ce qu'il en faut de moins en moins de temps pour les effectuer. Aider les joueurs à construire ce type de connaissances devient indispensable. Ainsi, si nous considérons la notion d'intervalle par exemple, comment peut-on passer d'une analyse statique à une analyse dynamique de celle-ci ? Dans une conception courante, l'intervalle est défini par la distance séparant deux joueurs à l'instant $\mathrm{T}$, voire un joueur et une limite de terrain (figure 1). Exploiter correctement l'existence de cet intervalle suppose d'avoir construit une vision dynamique de son évolution et surtout qu'au temps $\mathrm{T}+1$, il existe encore (Gréhaigne, 2009). Cela présume également d'envisager le contexte du jeu pour concevoir l'évolution de la configuration momentanée du jeu. Car au temps $\mathrm{T}+1$, cet intervalle peut rester identique si rien ne change (1). Il peut augmenter si les joueurs se sont écartés en fonction de l'évolution du contexte de jeu (2). Mais, il peut quasiment disparaître si l'évolution du jeu a entraîné une compression et qu'en conséquence les joueurs se sont rapprochés (3). En dehors de ces facteurs strictement statiques ou dynamiques, le déplacement d'un autre joueur, partenaire ou adversaire, peut venir fermer ou occuper cet intervalle dans un mouvement que l'on jugera judicieux ou intempestif en fonction du rapport d'opposition passager. 


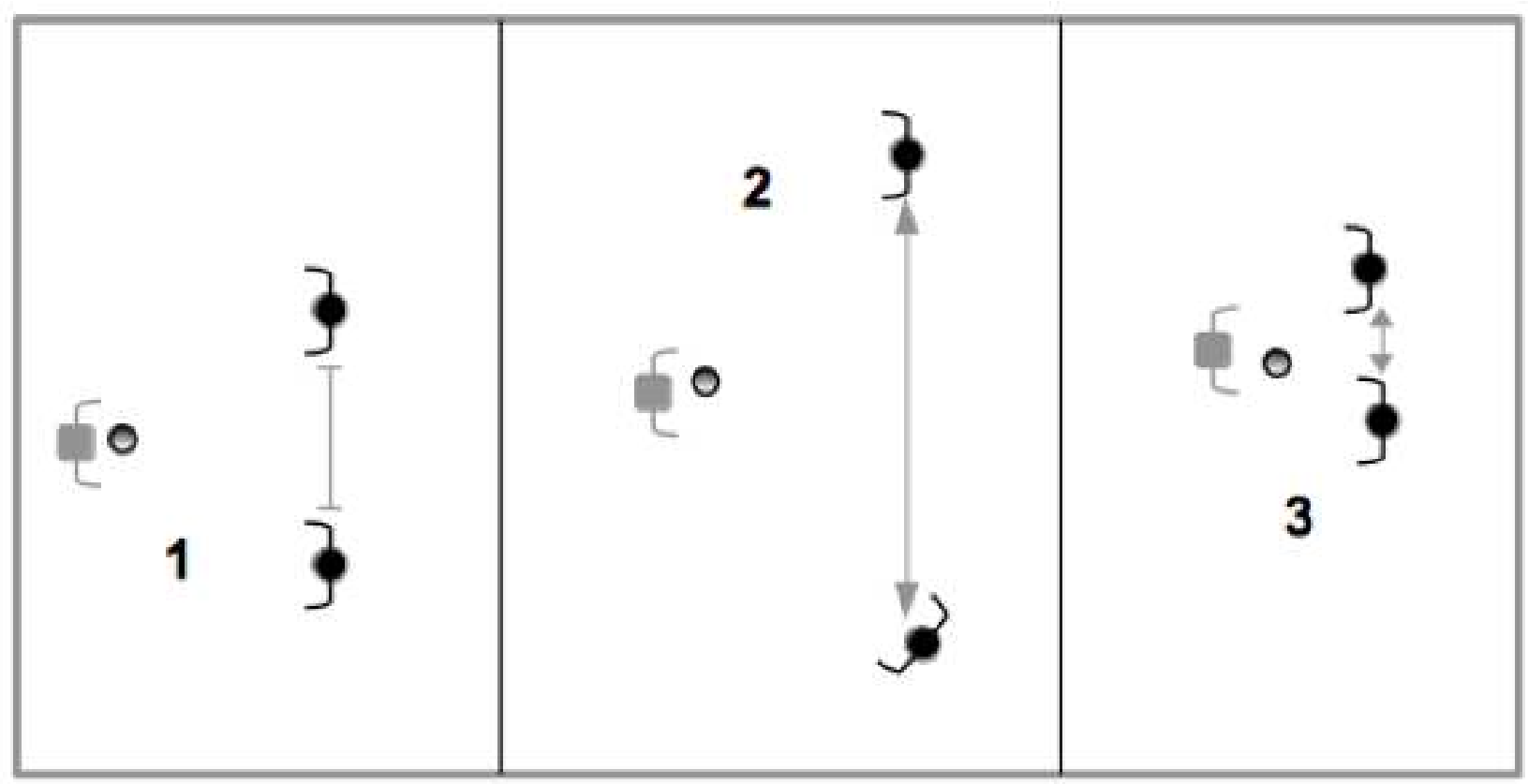

Figure 1. Dynamique des intervalles : trois cas de figure.

Il en est de même pour l'espace libre ou l'open space comme chez les anglophones. Une première distinction importante est à noter : utiliser l'espace libre ou créer l'espace libre ne renvoie pas aux mêmes opérations. Dans le premier cas, il préexiste et l'on peut l'exploiter. Dans l'autre cas, une circulation des joueurs est à mettre en place pour qu'il apparaisse. Une deuxième indication est fournie par les auteurs de littérature anglaise qui parlent d'espace ouvert (open space), signifiant bien que cet espace peut se refermer. Ici aussi, une vision statique ou dynamique de cet espace va donner lieu à des opérations différentes. L'espace libre est une donnée dynamique en perpétuelle évolution. Les courses, les déplacements des joueurs les font et les défont. On peut alors définir l'existence et la grandeur d'un espace libre à partir du temps que mettra un joueur donné pour le franchir et ceci à une vitesse donnée. Cette idée de distance à parcourir et de vitesse du joueur sont à notre sens les concepts clés. Par exemple, dans le cas d'une défense en ligne basée sur le hors-jeu, l'espace volontairement interdit par le placement des défenseurs crée un espace seulement utilisable dans des conditions restreintes, mais laisse aussi un espace potentiellement libre. Il devient alors un espace ouvert dans le dos des défenseurs si l'on sait l'utiliser.

Les aspects temporels du jeu nous semblent fondamentaux pour une bonne appréciation des rapports d'opposition. L'espace est un indicateur commode, mais le temps dont dispose un joueur ou un groupe de joueurs pour agir est à notre sens l'élément essentiel pour comprendre l'évolution d'un rapport de forces. 


\section{Contraction / expansion}

Ainsi envisagée, l'étude théorique des rapports d'opposition permet de dégager un certain nombre de concepts pertinents qui conduisent à la définition de variables ou d'indicateurs liés de façon plus ou moins compliquée (nombre, rapports, fonctionnalités) à des aspects observables. Une approche dynamique de l'affrontement (Gréhaigne \& Godbout, 2014) entraîne la nécessité d'utiliser des concepts qui rendent compte du mouvement continuel. À cet effet, et pour mieux analyser l'étalement du jeu en largeur et profondeur, nous avons présenté (Gréhaigne, 2009) les concepts de jeu expansion et / ou en contraction afin de constater ce que pourrait amener cette autre façon d'envisager l'analyse du rapport de forces entre deux équipes. Si nous poursuivons un peu plus avant notre réflexion, déplacements, contractions et expansions de l'espace de jeu effectif sont au cœur de la conception de notre modélisation. À un instant précis dans une rencontre, chaque équipe est caractérisée par un taux de désordre qui traduit le degré d'organisation ou de désorganisation de chaque groupe. Révélateur du fonctionnement collectif, ce taux doit néanmoins rester dans des valeurs acceptables pour permettre l'affrontement. Trop d'ordre apparent chez les attaquants impliquerait en effet une tactique aisée de récupération et de contre pour l'adversaire, tandis que trop de désordre induirait des aléas difficilement surmontables par les partenaires. Dans un affrontement partiel, l'interpénétration des sous-systèmes, et le fait qu'ils soient en barrage ou à la poursuite avec de l'avance pour l'attaque ou du retard sur le replacement défensif pour la défense, va façonner des rapports d'opposition spécifiques. Enfin, les trajets et les trajectoires de la balle fournissent des observables essentiels car en envoyant le ballon dans le dos des défenseurs par exemple, une bonne longueur de balle peut mettre l'adversaire en difficulté. Une accélération de la circulation du ballon, en diminuant le nombre de touches de balle entre les partenaires constitue, aussi, une option économique, fiable et sécuritaire de progression vers le but adverse (Dugrand, 1989 ; Lemoine \& Jullien, 2008). Dans un football moderne qui s'appuie fortement sur des stratégies défensives, le fait de marquer un but devient parfois difficile. En utilisant peu de touches de balle par joueur, les attaquants jouent plus vite et ainsi peuvent espérer contourner les défenseurs afin de pouvoir tirer au but (Lemoine, Jullien, \& Ahmaidi, 2005). Compression et extension pour le mouvement, contraction / expansion à propos d'états statiques successifs (ou des aspects temporels discontinus) semblent fonctionner en étroite symbiose pour permettre aux joueurs de décrypter et d'anticiper le mouvement dans le jeu et prendre alors des décisions judicieuses. Les principales actions concernant la circulation du ballon et des joueurs peuvent être ainsi inventoriées :

- balle arrêtée dans la contraction;

- balle arrêtée avec une phase d'expansion ;

- balle en mouvement vers ou dans la contraction ; 
- balle en mouvement dans une phase d'expansion.

Pour plus de précision, il faut bien sûr intégrer à cette analyse les trajets et trajectoires du ballon (dans l'axe ou à la périphérie) pour obtenir une appréciation plus exacte de la situation et du rapport de forces attaque / défense.

La succession des déformations de l'espace de jeu et l'évolution des configurations ainsi que l'élasticité inhérente aux formes du jeu constituent donc des indicateurs privilégiés de l'affrontement. Les joueurs ne sont plus conçus comme liés à un espace fixe, mais comme des mobiles occupant l'espace de jeu dans un cadre de potentialités (Fernandez, 2002).

\section{Jeu de transition et mouvement}

Le jeu de transition consiste en la conservation et la montée du ballon permettant une transition entre la récupération de la balle et la mise en place d'une attaque placée. La notion de transition est souvent confondue avec le passage du statut de défenseur au attaquant et vice et versa. Le jeu de transition renvoie, communément, à des configurations où l'on a un peu de temps pour agir (y compris pour la contre-attaque) : l'attaque essaie de conserver son avance éventuelle et la défense tente de revenir en barrage ou d'y rester. Le recours au jeu long, avec une frappe tendue, transforme vite les configurations du jeu, celles-ci évoluant, le plus souvent, vers un jeu en expansion. Le jeu en contraction existe, le plus couramment, sur une surface limitée du terrain avec une circulation de balle lente provoquant une densité importante de joueurs. Les transitions entre un jeu stabilisé dans sa zone de défense vers un jeu stabilisé dans la zone d'attaque sont très importantes à travailler et à analyser car on va souvent de la compression vers l'extension puis de l'extension vers la compression, obligeant à gérer des circulations tactiques très différentes. Ce serait donc, a priori, une référence essentiellement spatiale et non numérique qui fonctionnerait si l'on se réfère encore une fois à des états statiques. Néanmoins, il existe une relation simple entre dispersion, densité et distance : plus la distance entre les joueurs augmente, plus la densité diminue et plus la dispersion augmente jusqu'à une limite où elle deviendrait constante. Les limites de ces phénomènes seraient constituées, sur un continuum, par une répartition uniforme de tous les acteurs sur l'aire de jeu (correspondant à une dispersion maximale de ceux-ci) ou un regroupement en un seul endroit sur le terrain (correspondant à une concentration maximale de tous les joueurs). Dans la réalité, densité (variable plutôt qualitative) et dispersion / concentration (variables plutôt quantitatives) fonctionnent à l'intérieur de possibilités définies que sont les limites extérieures de l'aire de jeu utilisée. Dans une séquence de jeu, il peut exister au même moment une concentration dans certains endroits du terrain et une dispersion dans d'autres. Si l'on joue dans l'axe central, la dangerosité du mouvement en cours peut être appréciée en fonction du temps, des événements du jeu et de la proximité de la cible. Il en découle, par exemple, une concentration de joueurs dans l'axe but 
attaqué / but défendu et une dispersion importante à la périphérie du terrain. Une autre caractéristique de la circulation des joueurs est qu'elle peut être à la fois très rapide mais aussi entrecoupée d'une succession d'arrêts. Barrage / poursuite, position en avance / en retard, arrêt / mouvement deviennent des éléments premiers de la prise d'information.

\section{Ce qui se joue : le jeu de l'avance et du retard}

La rupture de l'équilibre attaque / défense avec effet de retard ou d'avance doit être conçue comme un point crucial de la tactique collective ainsi que la conservation de cette avance. Quand aucun déséquilibre existe, comment le faire apparaître ? Deux réponses simples consistent à amener rapidement le ballon en avant de l'espace de jeu ou à le récupérer en avant de cet espace. Deux autres réponses plus difficiles à mettre en œuvre consistent à attaquer dans la dimension momentanément faible de la défense ou à attaquer dans la dimension forte de la défense pour la transformer avec deux modalités typiques de prise d'avantage : la balle dans le dos et le jeu à la limite de la ligne de hors-jeu, ou des accélérations dans la prise d'intervalle. La première de ces modalités signifie la prise d'un adversaire (joueur ou groupement) en défaut par passage dans son dos. Il doit se retourner pour poursuivre son action avec le retard que constituent la coordination d'un passage en profondeur et l'amenée du ballon dans cette zone. Cette configuration de rupture touche à la protection d'une ligne d'avantage qui passe par le joueur ou la ligne défensive considérée. Le jeu " dans le dos" doit se situer dans un contexte plus large : celui de l'utilisation ou de la création d'espace libre à l'arrière du système défensif. Le jeu à la limite du hors-jeu contient une partie du jeu dans le dos, mais dans des conditions différentes. L'espace au-delà de la ligne de hors-jeu est toujours ouvert, mais sa liberté est régulée par des conditions d'utilisation (Fernandez, 2002).

Avec cette analyse temporelle, il faut bien différencier ce qui relève de «prendre de l'avance », de «utiliser l'avance». «Prendre de l'avance» consiste à mettre la défense en retard car pour l'instant le rapport de forces est équilibré. «Utiliser l'avance » consiste à conserver le temps d'avance préalablement acquis sur le replacement défensif par des choix judicieux.

\section{Ce qui se joue : le jeu en barrage ou à la poursuite}

11 apparaît indispensable que les élèves puissent décrypter rapidement quelles sont les dispositions spatiales et les vitesses des adversaires et des partenaires lors de la récupération du ballon. Quand une défense est en barrage ou en poursuite cela n'a pas la même signification ; les choix des tactiques et des compétences motrices ne seront pas identiques de la part des attaquants dans l'un ou l'autre cas. 
Une défense est en barrage si, globalement, elle est positionnée entre le ballon et son propre but. Quand une défense est en barrage, la possibilité de contre-attaque est quasiment inexistante. Les attaquants sont confrontés au fait de devoir mettre en place une attaque de position qui est une attaque lente face à une défense regroupée. On vise à bouger, à forcer, à déborder ou à fixer le bloc défensif adverse pour créer une avance temporelle permettant de tirer avec une chance de succès raisonnable. L'efficacité d'une attaque de position passe aussi par le développement d'une adaptation aux rapports d'opposition et des décisions justes.

Une défense est à la poursuite quand le porteur de balle se situe en avant de l'espace de jeu effectif avec les défenseurs derrière lui, ce que l'on retrouve bien souvent dans le cas d'une contre-attaque ou d'une récupération haute du ballon. Dans ces circonstances, la première mission des défenseurs concernés par la défense à la poursuite est de tenter de se replacer le plus vite possible entre le ballon et la cible. On voit bien que dans ce genre de recul fuite, le mouvement et la vitesse des joueurs sont premiers en vue de reconstituer le ou les rideaux défensifs.

A l'entraînement, il faut expérimenter ces types d'équilibres ou de déséquilibres (Zerai, Gréhaigne, $\&$ Godbout, 2013) en vue de construire des réponses disponibles. Ce type d'apprentissage « temporel » est important car ce sont des séquences de jeux que l'on retrouve fréquemment dans le jeu de plein mouvement.

\section{Temps et réponses du joueur}

A cette étape de notre développement, il est nécessaire de distinguer des opérations d'exécution (celles qui sont liées à la réalisation comme telle) et des opérations d'organisation (celles qui assurent le repérage de la situation et amène à une décision) en rapport avec les opérations cognitives du joueur. La base de connaissance de celui-ci consiste en l'ensemble de ses connaissances à propos de l'action elle-même aussi bien que les conditions dans lesquelles elle est accomplie. En d'autres termes, ce sont les représentations que le joueur possède à propos du jeu qui lui permettent de comprendre l'action en cours. L'approche, en terme de coût cognitif, consiste à considérer que les ressources cognitives sont limitées chez un individu donné, à un moment donné pour une tâche donnée. Or, toute tâche nécessite l'allocation d'une certaine quantité des ressources cognitives. Cette quantité varie en fonction du caractère plus ou moins automatique ou contrôlé des procédures mobilisées. Le facteur le plus influent en ce qui concerne la mobilisation des procédures semble être les connaissances et l'expérience du joueur. 


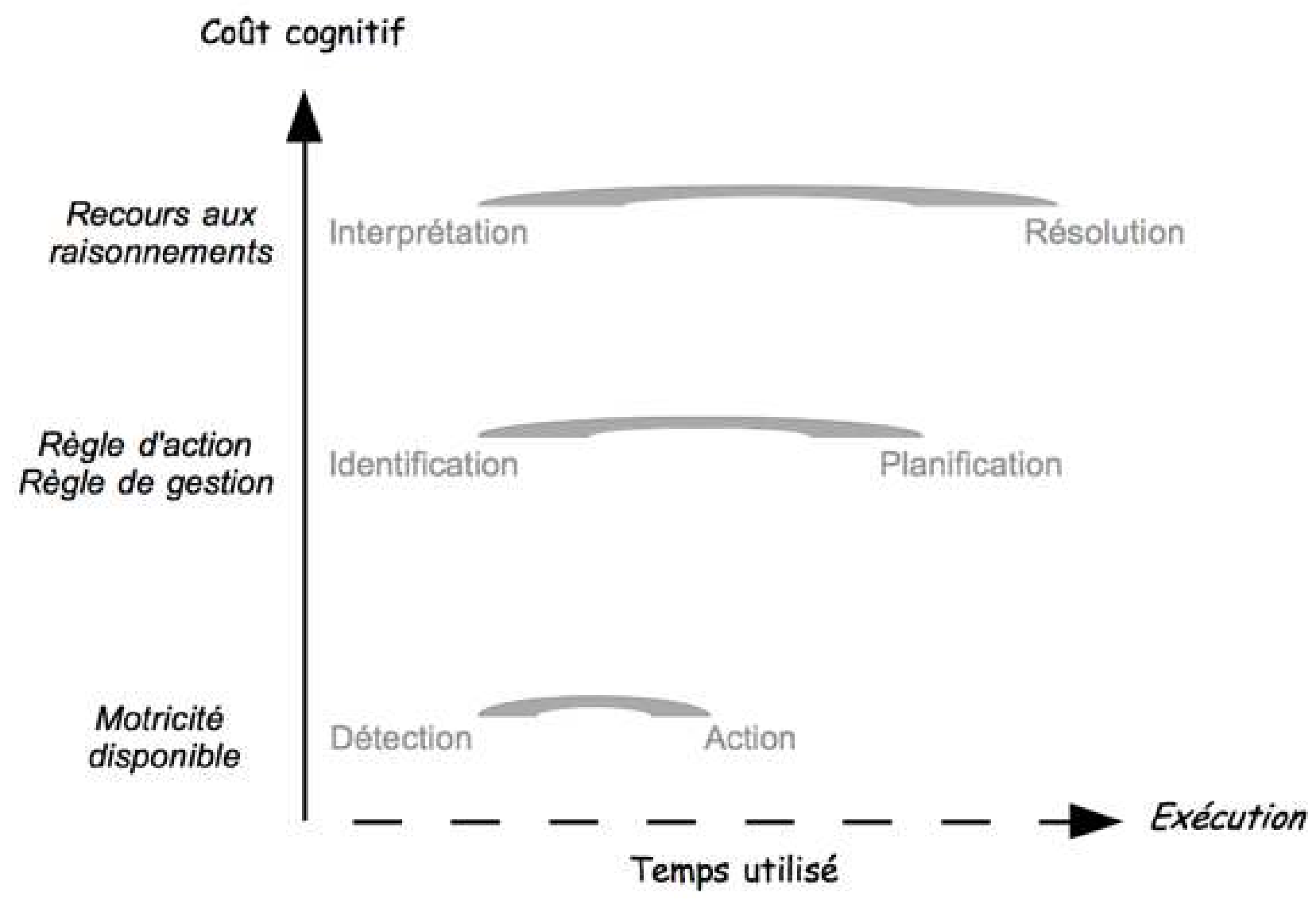

Figure 2. Organisation du jeu et contrôle dynamique.

La modélisation de l'organisation du jeu (figure 2) met en évidence un fonctionnement parallèle de trois boucles de réajustements qui s'inscrivent dans des échelles de temps et de coûts cognitifs différents (Hoc \& Amalberti, 1999). Par ailleurs, la hiérarchisation des opérations facilite, a priori, l'efficacité de l'analyse de la tâche en vue de construire une anticipation sur ce qui devra être effectué, ainsi qu'une planification des activités à mettre en œuvre.

On distingue trois niveaux différents de l'activité d'élaboration d'une réponse, avec des coûts temporels très divers. Dans le premier niveau de traitement de l'information, les opérations sont le plus souvent traitées en tâche de fond par le joueur. Elles s'appuient sur la détection de signaux, de repères et d'indices qui orientent immédiatement le joueur vers la réponse appropriée, sans passer par la représentation symbolique. Le deuxième niveau de traitement de l'information concerne les situations de jeu où les stimuli et repères ne sont plus exploités au niveau de leurs caractéristiques physiques, mais au niveau du contenu qu'ils véhiculent. Les signaux ne sont plus aussi clairs pour les activités évaluatives qui doivent laisser place à une interprétation de la situation par le joueur. Celle-ci se fonde sur sa compréhension du jeu à l'aide des règles d'action et des règles de gestion de l'organisation du jeu qu'il maîtrise en vue d'une planification adaptée (Gréhaigne, Poggi, \& Zerai, 
2017). Le troisième niveau de traitement de l'information renvoie à des mécanismes interprétatifs encore plus profonds et plus longs à mettre en œuvre que la simple orientation vers un ensemble de règles d'action. Les repères sont plus subtils et se basent sur une interprétation encore plus détaillée du jeu. Le niveau de contrôle des actions par le joueur renvoie à la notion de résolution de problèmes où la durée de traitement devient un facteur important.

Ainsi, dans un environnement dynamique comme dans les sports collectifs, la compréhension de l'état en cours du jeu et l'anticipation des actions des partenaires et des adversaires vont de paire pour assurer un contrôle optimal des décisions et des actions.

\section{Conclusion}

Avec les progrès dans le jeu et l'élévation du niveau, la reconnaissance et l'exploitation des configurations momentanées du jeu sont à effectuer dans des rapports d'opposition de plus en plus complexes. La complexité repose ici sur le temps et la vitesse à disposition pour décider et faire. Temps et vitesse deviennent des éléments cruciaux de la réussite et le nécessaire cadre de référence à toute évaluation formative. Le temps et l'espace constituent un système, mais cette façon de poser les aspects temporels comme premiers devrait permettre de passer de «j'ai de la place » à «j'ai le temps de passer la balle » ou «j'ai le temps de me déplacer avec le ballon ». En fonction des configurations perçues du jeu, les rapports de vitesse deviennent prégnants, le démarquage et l'espace libre ne constituant alors que des conséquences du facteur temps : le temps dont dispose chaque joueur pour conserver ou perdre son avance en fonction des autres. Il faut tenir compte aussi qu'avec une durée importante de la circulation des joueurs et du ballon, une séquence de jeu devient de moins en moins prédictible, car des désordres se sont installés de façon inéluctable compliquant la prise d'informations et la compréhension de l'évolution du jeu en vue d'apporter des réponses judicieuses pour les joueurs.

En définitive, rien ne peut être fondamentalement compris en sport collectif si l'on ne quitte pas un référentiel spatial pour un référentiel temporel permettant de traiter de façon plus pertinente les informations à disposition. En sport collectif, l'espace n'est qu'un indicateur, certes privilégié, d'un rapport d'opposition. Toutefois, le temps dont dispose un joueur ou un groupe de joueurs pour agir est à notre sens l'élément fondamental majeur pour comprendre et faire évoluer le jeu.

Pour le joueur en formation, doit-on mettre l'accent sur « ai-je le temps » ou « ai-je l'espace » pour jouer le bon coup que je viens de décider de faire ? Certains collègues pensent que le détour par l'espace est nécessaire pour construire les concepts de temps d'avance ou de durée. Alors, comment s'y prend-on dans une didactique incluant le temps ? Cela recouvre quelles connaissances pour le maître et / ou pour l'élève pour enseigner ou apprendre des données temporelles ? 
Ces interrogations restent plus que jamais d'actualité... et nous allons y revenir dans le chapitre suivant.

\section{Bibliographie}

Bergson, H. (1922). Durée et Simultanéité. À propos de la théorie d'Einstein. Paris : Presses Universitaires de France

Deleplace, R. (1979) Rugby de mouvement - Rugby total. Paris : Éducation Physique et Sports.

Dugrand, M. (1989). Le football : de la transparence à la complexité. Paris : PUF.

Fernandez, A. (2002). Rupture et prise d'avantage en sports collectifs, un modèle théorique. eJournal de la Recherche sur l'Intervention en Education Physique et Sportive, 2, 03-17.

Gréhaigne, J.-F., Godbout, P., \& Bouthier, D. (1997). Performance assessment in team sport. Journal of Teaching in Physical Education ,16, 500-516.

Gréhaigne, J.-F. (Ed.). (2009). Autour du temps. Espaces, apprentissages, projets dans les sports collectifs. Besançon : Presses de l’Université de Franche-Comté.

Gréhaigne, J.-F., \& Godbout, P. (2012). À propos de la dynamique du jeu... en football et autres sports collectifs. eJRIEPS, 26, 130-156.

Gréhaigne, J.-F., \& Godbout, P. (2014). Dynamic systems theory and team sport coaching Quest, 66 (1), 96-116.

Gréhaigne, J-F., Poggi,M-P., \& Zerai, Z.(2017). Connaissances et compétences motrices en sport collectif : quoi enseigner ? eJRIEPS, 40, 143-162.

Hoc, J.-M., \& Amalberti, R . (1999). Analyse des activités cognitives en situations dynamiques : D'un cadre théorique à une méthode. Le Travail Humain, 62, 97-129.

Lemoine, A., \& Jullien, H. (2004). Etude de la production d'Information dans le cadre de la transmission instantanée du ballon en football. eJRIEPS, 6, 47-55.

Lemoine, A., Jullien, H., \& Ahmaidi, S. (2005). Technical and tactical analysis of one-touch playing in soccer - Study of the production of information', International Journal of Performance Analysis in Sport, 5: 83-103.

Zerai, Z., Gréhaigne, J.-F., \& Godbout, P. (2013). Configurations of play in invasion team sports and learning by analogy. International Journal of Physical Education Volume L (1) 1828. 